\title{
Guided growth for correction of knee flexion deformity: a series of four cases
}

\author{
B. A. MacWilliams • B. Harjinder • \\ P. M. Stevens
}

Received: 1 October 2010/Accepted: 5 July 2011/Published online: 22 July 2011

(C) The Author(s) 2011. This article is published with open access at Springerlink.com

\begin{abstract}
Fixed knee flexion deformity can present as an insidious and significant problem in diverse etiologies, most commonly in cerebral palsy. Traditional surgical intervention has included posterior capsulotomy and supracondylar femoral osteotomy, both of which carry significant associated morbidity and risks. In the skeletally immature patient, guided growth may be used to correct or substantially diminish the deformity. We are presenting our early experience encompassing four subjects who completed instrumented gait analysis both prior to and after distal femoral anterior guided growth (hemiepiphysiodesis). Changes in gait and function resulting from surgery in each individual are reported. Outcomes indicate improved knee range of motion and alleviation of crouch at the knee with secondary improvements in the ankle, hip and pelvis. Three subjects with initially slow gait velocity improved to within normal limits by demonstrating increased stride length. A measure of overall gait kinematics showed improvements in all limbs. Anterior guided growth (hemiepiphysiodesis) of the distal femur resulted in positive quantitative changes in all four patients, though degree and types of changes were variable in this small series. Encouraged by these findings, we now prefer guided growth to extension supracondylar
\end{abstract}

B. A. MacWilliams $(\bowtie) \cdot$ B. Harjinder

Motion Analysis Laboratory, Shriners Hospitals

for Children-Salt Lake City, Fairfax Rd. @ Virginia St.,

Salt Lake City, UT 84103, USA

e-mail: bmacwilliams@shrinenet.org

B. A. MacWilliams · P. M. Stevens

Department of Orthopaedic Surgery, University of Utah,

Salt Lake City, UT, USA

P. M. Stevens

Primary Children's Medical Center, Salt Lake City, UT, USA osteotomy for the skeletally immature patient with fixed knee flexion deformity.

Keywords Fixed knee flexion deformity - Crouch gait . Guided growth $\cdot$ Hemiepiphysiodesis $\cdot 8$-plate

\section{Introduction}

Fixed knee flexion deformity (FKFD) can present as an insidious and significant problem in diverse etiologies like $\mathrm{CP}$, arthrogryposis and myelomeningocele, with $\mathrm{CP}$ being relatively more common [1-3]. Conservative management including physical therapy and bracing, often combined with Baclofen or selective and temporary chemical denervation of muscles using botulinum toxin or phenol may be prescribed to address more mild presentations $[4,5]$. However, the natural progression of the impairment is from muscle spasticity to contracture and eventually bony deformity [6]. Co-spasticity of muscles acting across joints and the development of musculoskeletal deformities make gait more laborious and energy consuming [7].

Surgical options after failure of conservative management include multi-level soft tissue and bony procedures, bipolar frame distraction, supracondylar osteotomy, anterior distal femoral hemiepiphysiodesis or guided growth $[6$, $8-12]$. The effect of supracondylar extension osteotomy is neutralized at a rate of about $1^{\circ}$ per month by rapid growth; recurrence has also been reported following frame distraction $[13,14]$.

The benefits of gradual correction of FKFD by guided growth using the flexible construct of the 8-plate has been documented in a previous article by the senior author (PMS) [15]. This article focuses on gait analysis data that include physical examination for a comprehensive 
assessment of outcome. This is the first report that utilizes gait analysis to objectively assess correction of FKFD after anterior distal femoral guided growth.

\section{Materials and methods}

Four patients, all men, with a diagnosis of FKFD secondary to an underlying neurological or congenital disorder participated in this prospective IRB-approved study. Subject demographics are given in Table 1, and a preoperative radiograph of subject 1 is provided in Fig. 1. A standard marker model was applied, and data were collected using a ten camera motion capture system (Vicon, Centennial CO, USA) and four force platforms (AMTI, Watertown, MA, USA) as the subjects ambulated at a self-selected velocity along a $10-\mathrm{m}$ walkway [16]. A standard physical examination by an experienced therapist was performed to measure passive range of motion at the knee, hip and ankle. The Gross Motor Function Measure (GMFM) Questionnaire sections $\mathrm{D}$ and $\mathrm{E}$ (standing/balance and walking) [17, 18] and the Gillette Functional Assessment Questionnaire (FAQ) [19] were administered to families, and the patients' Gross Motor Function Classification System [20] was rated by a physical therapist. Within 1 month following the preoperative gait study, each subject subsequently underwent anterior distal femoral guided growth, placing a plate on either side of the patello-femoral sulcus.

\section{Technique}

Under tourniquet control, using fluoroscopic guidance, the distal femoral physis was identified; this is best observed in the lateral, cross-table projection. Two incisions about $3 \mathrm{~cm}$ long were made on either side of the patella, centered over the physis. The capsule and synovium were opened, the femoral sulcus visualized, and the plates placed just outside the articular portion of the joint surface. The central hole of the 8-plate was slipped over the Keith needle, which was inserted into the physis. Two $1.6-\mathrm{mm}$ guide wires were then passed through the proximal and distal

Table 1 Subject demographics

\begin{tabular}{lllll}
\hline Subject & Diagnosis & Side(s) & Age at pre & $\begin{array}{l}\text { Implant time } \\
\text { (months) }\end{array}$ \\
\hline 1 & Diplegia & Bilateral & $11(5)$ & 24 \\
2 & Arthrogryposis & Bilateral & $12(9)$ & 12 \\
3 & Diplegia & Bilateral & $14(2)$ & 13 \\
4 & CDP & Left & $9(5)$ & 29 \\
\hline
\end{tabular}

Age is given in years (months)

$C D P$ congenital dislocated patellae

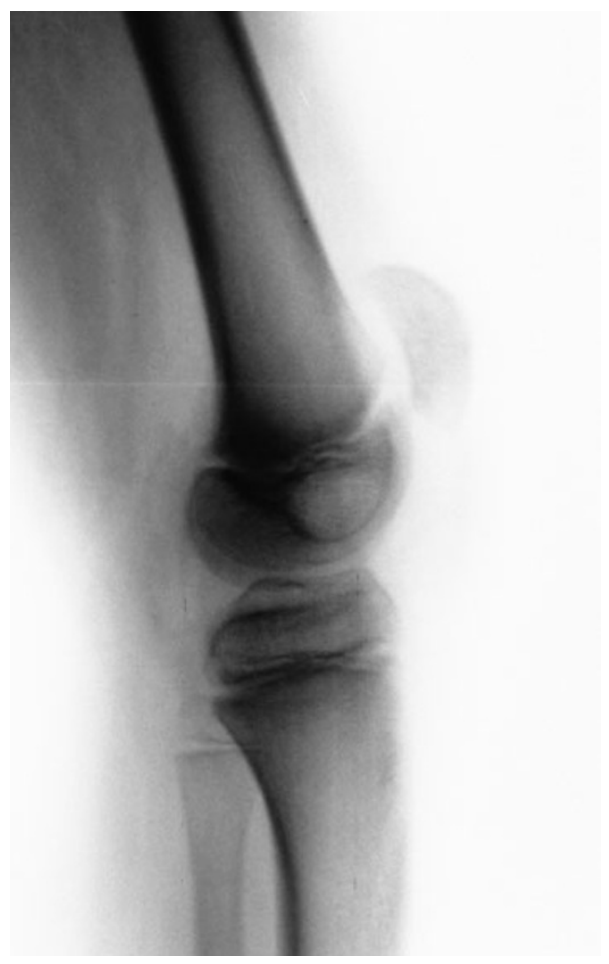

Fig. 1 Bilateral fixed knee flexion deformity of $18^{\circ}$ causing obligatory crouch gait and associated anterior knee pain (subject 1)

holes of the plate under fluoroscopic guidance, taking care not to violate the physis or the joint. The cortex was drilled (5 mm), and self-tapping, cannulated, 24-mm screws were inserted. Upon removal of the guide pins, the screws were further tightened to countersink them within the plate. The screws need not be parallel, and the implants were intracapsular, but not on the articular surface (Fig. 2). The wound was closed, soft dressing applied, and the patient was allowed ambulation as tolerated. Follow-up films were taken at 4 weeks to assure the position and integrity of the plates (Fig. 3). The patients were followed at 3-6 month intervals. When full passive extension was achieved, the gait analysis study and physical examination were repeated. Within 1 month following the gait study, plates were removed (average plate insertion time 15.07 months, range 11-23 months).

Data analysis

Due to the small number of participants in this study, data are presented individually. Gait analysis measures focus on key sagittal plane parameters during stance. Additionally, an overall measure of gait kinematics is reported using the gait deviation index [21]. Two physical examination measures of the knee flexion contracture and popliteal angle are also provided. Using all treated limbs, means and confidence intervals are presented for the following 


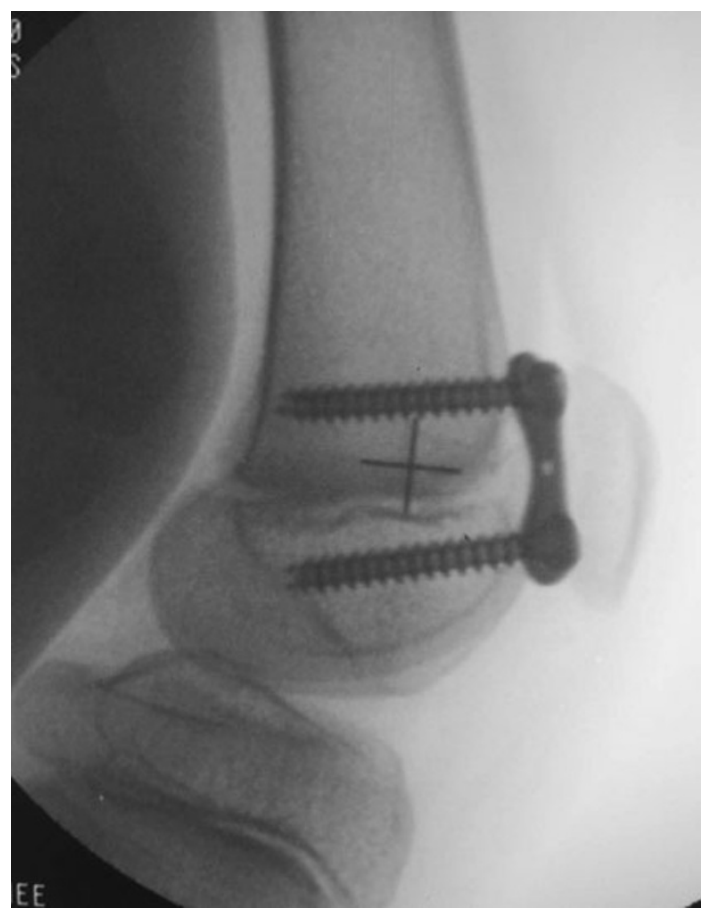

Fig. 2 Intraoperative fluoroscopic view demonstrating the positioning of the anterior 8-plate (subject 1), one is placed on each side of the patello-femoral sulcus. They are intracapsular but non-articular

kinematic variables: knee and hip flexion at initial contact, maximum knee and hip extension in stance, and GDI. Gait data from a typically developing group of similar aged
(8-12 year old $)$ children $(n=38)$ collected from a separate IRB-approved study are given for comparison.

\section{Results}

In this study, varying degrees of improvement in both the fixed deformities as measured during physical examination and by sagittal knee kinematics during gait were determined. Degree of knee flexion contracture and popliteal angle from physical examination are reported in Table 2; velocity, cadence and stride length are reported in Table 3. Complete sagittal plane gait data are presented in Fig. 4 with statistical evaluation of group results of key measures presented in Fig. 5. Results are summarized first individually then by group.

\section{Subject 1}

In subject 1 , an individual with diplegic $\mathrm{CP}$, no change in the left side and only a slight improvement in fixed knee flexion deformity on the right side were observed by physical examination; yet, this subject experienced a complete correction according to his gait curves. His GDI indicated that his gait returned to within normal control values, an improvement in more than two standard deviations. He did not improve temporally or functionally, but was normal in these measures prior to surgery. Thus, in this
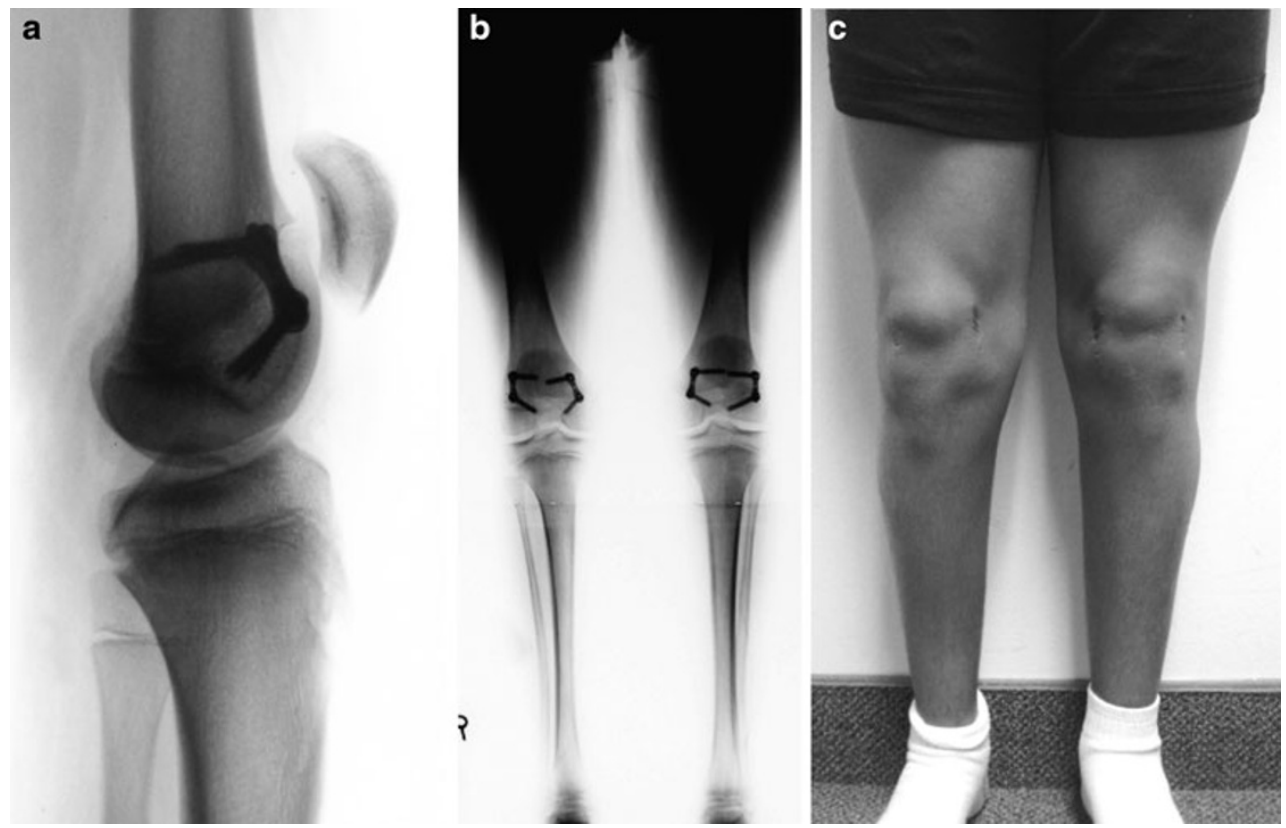

Fig. 3 a Lateral view documenting full correction of the fixed knee flexion deformity. Despite the residual patella alta, he has no pain, patellar instability, or related functional limitations; therefore, the patellar tendon does not need to be advanced. b A full-length radiograph showing the retained 8-plates. c Clinical appearance at the time of follow-up gait analysis. Upon full correction of the fixed knee flexion deformity, the 8-plates are typically removed. All images are from subject 1 
Table 2 Physical examination results and gait deviation index (GDI)

\begin{tabular}{|c|c|c|c|c|c|c|c|}
\hline \multirow[t]{2}{*}{ Subject } & \multirow[t]{2}{*}{ Side } & \multicolumn{2}{|c|}{ Knee flexion } & \multicolumn{2}{|c|}{ Popliteal angle } & \multicolumn{2}{|l|}{ GDI } \\
\hline & & Pre & Post & Pre & Post & Pre & Post \\
\hline \multirow[t]{2}{*}{1} & $\mathrm{~L}$ & 7.5 & 7.5 & -35 & -40 & 79.8 & 101.9 \\
\hline & $\mathrm{R}$ & 10 & 7.5 & -30 & -40 & 68.7 & 101.3 \\
\hline \multirow[t]{2}{*}{2} & $\mathrm{~L}$ & 15 & 0 & -45 & -45 & 48.6 & 65.2 \\
\hline & $\mathrm{R}$ & 15 & 0 & -40 & -45 & 47.4 & 50.5 \\
\hline \multirow[t]{2}{*}{3} & $\mathrm{~L}$ & 22.5 & 10 & -60 & -50 & 56.2 & 65.9 \\
\hline & $\mathrm{R}$ & 20 & 0 & -65 & -45 & 62.2 & 74.7 \\
\hline 4 & $\mathrm{~L}$ & 27.5 & 0 & -35 & -30 & 48.0 & 72.1 \\
\hline Ave & & 16.8 & $3.6^{*}$ & -44.3 & -42.1 & 58.7 & $75.9 *$ \\
\hline
\end{tabular}

GDI is a normalized score determined from nine kinematic variables where values $>90$ are within one standard deviation of an age-matched control set, and each 10 points represents differences of one standard deviation

Popliteal angle is measured from the vertical reference

*Significantly different changes from pre- to post-analysis

Table 3 Temporal-spatial gait parameters and functional tests

\begin{tabular}{|c|c|c|c|c|c|c|c|c|c|c|c|c|}
\hline \multirow[t]{2}{*}{ Subject } & \multicolumn{2}{|c|}{ Velocity (m/s) } & \multicolumn{2}{|c|}{ Cadence (steps/s) } & \multicolumn{2}{|c|}{ Stride length (m) } & \multicolumn{2}{|c|}{ GMFCS } & \multicolumn{2}{|c|}{ GMFM-66 } & \multicolumn{2}{|c|}{ FAQ } \\
\hline & Pre & Post & Pre & Post & Pre & Post & Pre & Post & Pre & Post & Pre & Post \\
\hline 1 & 1.25 & 1.04 & 1.06 & 0.92 & 1.18 & 1.13 & 1 & 1 & 100 & 100 & 10 & 10 \\
\hline 2 & 0.86 & 1.13 & 1.09 & 1.10 & 0.79 & 1.02 & 3 & 2 & 63 & 67 & 9 & 9 \\
\hline 3 & 0.63 & 1.19 & 1.01 & 1.02 & 0.62 & 1.17 & 1 & 1 & 85 & 78 & 8 & 9 \\
\hline 4 & 0.97 & 1.23 & 1.01 & 1.01 & 0.96 & 1.22 & 1 & 1 & 100 & 100 & 10 & 10 \\
\hline
\end{tabular}

Velocity is measured in meters per second, cadence in steps per second and stride length in meters

GMFCS Gross Motor Function Classification System, GMFM-66 66-item normalized Gross Motor Function Measure sections D and E, FAQ Gillette Functional Activity Questionnaire

case, the underlying neurological deficit was mild, and a slight correction of the bony deformity enabled a normal walking pattern.

\section{Subject 2}

In subject 2, an individual with arthrogryposis, large changes in fixed knee deformity $\left(15^{\circ}\right.$ bilaterally) were documented, and his gait pattern was changed from crouch gait to back-kneeing. This subject made significant gains in velocity, and improved his GMFCS from a level 3 to a level 2 ambulator as he was able to walk without use of a crutch after surgery, though GMFM-66 and FAQ scores were stable. In this case, correction of the FKFD may have unmasked underlying quadriceps weakness, resulting in the recurvatum. This can, however, be controlled by orthoses and is considered a good outcome. Physical examination revealed that ankle range of motion did not change, remaining at a maximal dorsiflexion of neutral with the knee extended or flexed. Post-surgical kinetics revealed reduced ankle moment support, indicating that recurvatum did not result from an increased ankle plantarflexion-knee extension couple. Radiographs indicate vertical tali, which may contribute to persistent abnormal ankle kinematics.

\section{Subject 3}

In subject 3, while FKFD improved markedly by physical examination $\left(12.5^{\circ}\right.$ on the left and $20^{\circ}$ on the right), these improvements translated into more minor gains in crouch gait compared with subject 1 . Overall gait kinematics improved by one standard deviation, considering a clinically significant gain [22], and were most obviously realized in improved pelvis and trunk positions. The subject had large temporal improvements, as velocity nearly doubled and returned to within normal limits with gains resulting from improvement in stride length. This subject's function decreased after surgery as GMFM-66 decreased by seven points due to a poorer performance in standing and balance (85\%) in contrast to walking (94\%). In particular, this subject decreased in ability to balance on a single leg and rise from the floor. This might be attributed to this subject's autism and ADHD and related pharmaceutical treatment. Despite the loss in balance, walking 

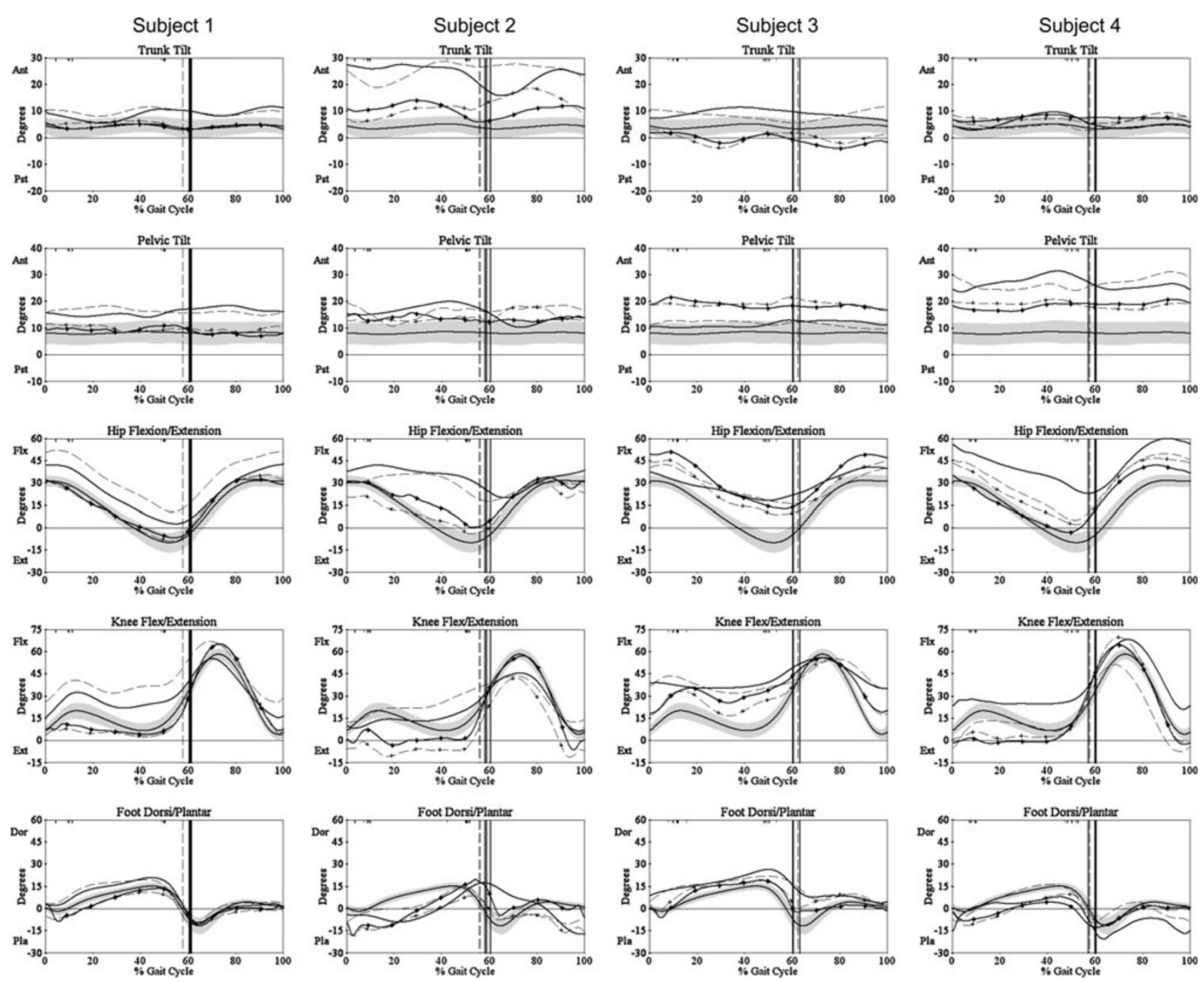

Fig. 4 Sagittal kinematics during gait of each subject. Each line represents an average of five trials. Preoperative data have no symbol, and postoperative data are starred. Left side data are solid, and right
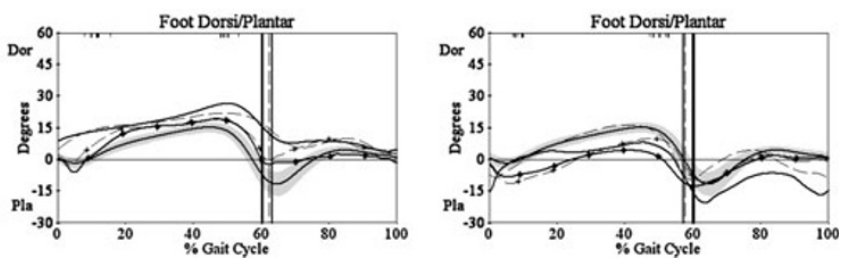

side data are dashed. Gray solid line and shaded band indicate means and one standard deviation from a pediatric control group

ability according to his FAQ score improved from 8-9. Additionally, at a later evaluation (4 years following surgery), his GMFM-66 score increased to 92, indicating a gain from his preoperative study. This subject also complained of significant anterior knee pain prior to surgery, which was resolved.

\section{Subject 4}

Subject 4 was the only unilateral subject, having bilateral congenital dislocated patellae, but knee flexion contracture and epiphysiodesis of the left limb only. This subject's knee contracture improved from $27.5^{\circ}$ to $0^{\circ}$ and resulted in alleviation of his crouch gait pattern, though a straight leg stance pattern resulted. Like subject 2, there was no kinetic evidence of an ankle plantarflexion-knee extension couple.

This subject's velocity and stride length improved to within normal limits, and GDI improved more than two standard deviations.

Comparisons of key variables on the seven limbs studied revealed significant alleviation of the crouch gait imposed by FKFD.

Physical examination

Knee flexion contractures improved in all but one limb, and as a group, changes were statistically significant (Table 2 , $P=0.027)$. Popliteal angles improved in the subject with the greatest degree of contracture (subject 3), but were otherwise variable, and not statistically significant $(P=$ $0.63)$. It is noteworthy that this technique does not produce the loss of knee flexion that is typically seen after posterior 

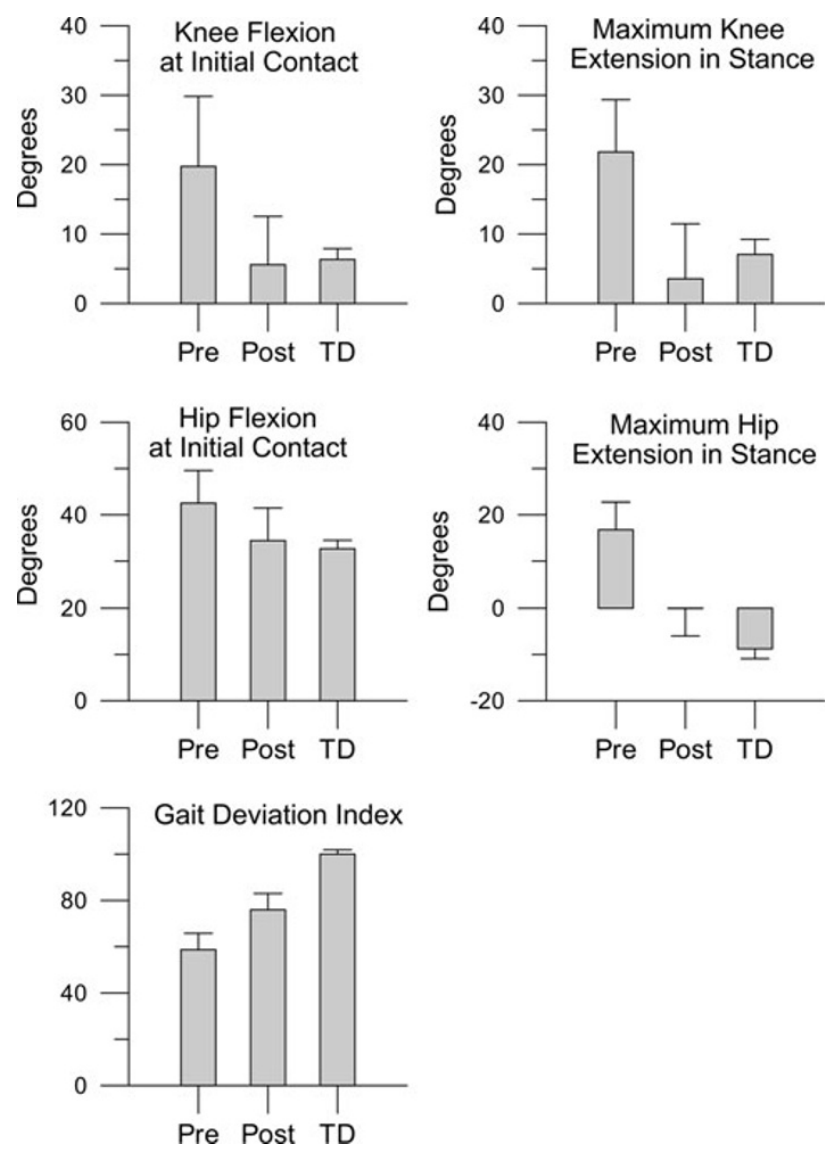

Fig. 5 Means of selected key kinematic variables to assess crouch gait profile and GDI for the seven limbs studied. Error bars represent the $95 \%$ confidence interval. Typically developing (TD) data are given to refer the changes from surgery

release of supracondylar osteotomy. In fact, the arc of motion is effectively increased.

Temporospatial and standardized functional measures

Temporal measures improved to within normal limits $(1.26 \pm 0.28 \mathrm{~m} / \mathrm{s})$ with surgery in subjects 2,3 and 4 with increases in velocity resulting from increases in stride length (Table 3). Velocity in subject 1 decreased, but as a result of reduced cadence, it was within normal limits at both evaluations. GMFCS level improved in subject 2 from a level 3 to level 2, but decreased in subject 3 from a level 1 to level 2 .

\section{Kinematics}

Sagittal knee curves were completely normalized in subject 1 (Fig. 4). Secondary to this improvement, trunk, pelvis, hip and ankle also improved to within normal limits. In subjects 2 and 4, crouch was alleviated but transformed to a recurvatum knee pattern. In subject 3, gains in knee extension at initial contact were apparent, but less dramatic throughout stance phase. Marked improvements were noted throughout stance at the trunk, pelvis and ankle. The gait deviation index improved in all sides of all patients, with values of subject 1 improving to within control limits $(>90)$.

Analysis of the seven limbs indicated that both knee flexion at initial contact and maximum knee extension in stance values both improved reflecting the relief of crouch gait (Fig. 5). At the hip, extension at initial contact decreased indicating less compensation necessary to achieve adequate step length. Maximal hip extension improved reflecting relief of crouch gait. In all limbs, each of these variables that were initially outside the normal range ( \pm 1 standard deviation) showed improvement. Those variables that were initially within normal range remained within normal range.

\section{Discussion}

Knee flexion contractures significantly alter the kinematics of walking and dramatically reduce the comfort and efficiency of ambulation. Co-spasticity of muscles acting across joints and the development of musculoskeletal deformities make gait more laborious and energy consuming [23]. This also increases the load on the hip and knee joints. Progressive flexion places more force on the quadriceps, leading to overstretching of the muscle fibers and the infrapatellar tendon, causing patella alta, patellar fragmentation, chondromalacia, joint instability, muscle weakness in terminal extension and pain secondary to patellofemoral degenerative joint disease [24, 25]. The benefits of guided growth for FKFD have been recently documented [15].

This minimally invasive technique is well tolerated by an already-compromised population. Our current preference is to offer distal femoral guided growth to address the fixed knee deformity. We no longer favor the aggressive approach of distal femoral osteotomy/patellar tendon advancement in the growing patient. Because these patients have experienced excellent relief of knee pain and improved gait, we have accepted patella alta as a radiographic finding without clinical significance once full knee extension has been restored. Likewise, we do not recommend concomitant hamstring recession, deferring this decision until the plates are removed. Parents and physical therapists are counseled preoperatively that, although the incisions are small and immediate ambulation is permitted, children may experience some pain and crepitance around the anterior plates that are just beneath the extensor retinaculum. These symptoms generally abate within 6-12 weeks. If the deformity recurs after hardware removal, the procedure is easily repeated.

This is but a small sample of patients at our institutions who have undergone distal femoral guided growth to 
correct fixed knee flexion deformity. Gait analysis is not routinely ordered for these individuals as their primary orthopedic problem is obvious. This report focuses on just four patients who did undergo both pre- and postoperative gait analysis.

This report focuses on six key variables that most directly measure the effects of anterior guided growth for knee contraction. The physical examination measure of knee flexion contracture by goniometer is presented along with five variables from instrumented gait analysis. Each of the gait variables that were initially abnormal improved in each patient with surgery. Knee flexion contractures measured by a physical therapist improved in all but one subject limb. It is likely that the initial measures in this subject were inaccurate as radiographically $18^{\circ}$ of contracture was initially determined, and the surgeon determined $20-25^{\circ}$ of contracture bilaterally. This subject also showed marked improvements in gait parameters following surgery. A residual knee contracture due to hamstring tightness may have been present postoperatively and is supported by worsening in popliteal angles recorded. Dynamic loading during gait may create moments about the ankle and knee sufficient to overcome this latent contracture as measured during passive range of motion examination.

In this preliminary study of outcomes of four patients, improvements were found in all patients following guided growth to correct fixed knee flexion deformity. Improved range of motion at the knee markedly improved stride length and self-selected velocity, and alleviation of the crouch gait pattern is reported. Along with improvement in knee kinematics during ambulation, secondary improvements at the hip, pelvis and trunk are also noted. Increases in velocity and stride length occurred in the three subjects (2, 3 and 4) who had presented with less than normal values preoperatively. Though specific changes are somewhat diverse, all four cases are viewed as good or excellent outcomes.

Acknowledgments Shriners Hospital donated the use of the movement analysis laboratory.

Conflict of interest No other financial support was received for this study.

Open Access This article is distributed under the terms of the Creative Commons Attribution License which permits any use, distribution and reproduction in any medium, provided the original author(s) and source are credited.

\section{References}

1. Mielke CH, Stevens PM (1996) Hemiepiphyseal stapling for knee deformities in children younger than 10 years: a preliminary report. J Pediatr Orthop 16:423-429
2. van der Krogt MM, Doorenbosch CA, Harlaar J (2007) Muscle length and lengthening velocity in voluntary crouch gait. Gait Posture 26:532-538

3. Wren TA, Rethlefsen S, Kay RM (2005) Prevalence of specific gait abnormalities in children with cerebral palsy: influence of cerebral palsy subtype, age, and previous surgery. J Pediatr Orthop 25:79-83

4. Carbonell PG, Valero JV, Fernandez PD, Vicente-Franqueira JR (2007) Monolateral external fixation for the progressive correction of neurological spastic knee flexion contracture in children. Strategies Trauma Limb Reconstr 2:91-97

5. Molenaers G, Desloovere K, Fabry G, De Cock P (2006) The effects of quantitative gait assessment and botulinum toxin a on musculoskeletal surgery in children with cerebral palsy. J Bone Joint Surg Am 88:161-170

6. Bache CE, Selber P, Graham HK (2003) The management of spastic diplegia. Current Orthopaedics 17:88-104

7. Saraph V, Zwick EB, Zwick G, Steinwender C, Steinwender G, Linhart W (2002) Multilevel surgery in spastic diplegia: evaluation by physical examination and gait analysis in 25 children. J Pediatr Orthop 22:150-157

8. Carbonell PG, Valero JV, Fernandez PD, Vicente-Franqueira JR (2007) Monolateral external fixation for the progressive correction of neurological spastic knee flexion contracture in children. Strategies Trauma Limb Reconstr 2:91-97

9. Drummond DS, Rogala E, Templeton J, Cruess R (1974) Proximal hamstring release for knee flexion and crouched posture in cerebral palsy. J Bone Joint Surg Am 56:1598-1602

10. Keats S, Kambin P (1962) An evaluation of surgery for the correction of knee-flexion contracture in children with cerebral spastic paralysis. J Bone Joint Surg Am 44:1146-1154

11. Molenaers G, Desloovere K, Fabry G, De Cock P (2006) The effects of quantitative gait assessment and botulinum toxin a on musculoskeletal surgery in children with cerebral palsy. J Bone Joint Surg Am 88:161-170

12. Saraph V, Zwick EB, Zwick G, Steinwender C, Steinwender G, Linhart W (2002) Multilevel surgery in spastic diplegia: evaluation by physical examination and gait analysis in 25 children. J Pediatr Orthop 22:150-157

13. Asirvatham R, Mukherjee A, Agarwal S, Rooney RJ, Ellis RD, Watts HG (1993) Supracondylar femoral extension osteotomy: its complications. J Pediatr Orthop 13:642-645

14. DelBello DA, Watts HG (1996) Distal femoral extension osteotomy for knee flexion contracture in patients with arthrogryposis. J Pediatr Orthop 16:122-126

15. Klatt J, Stevens PM (2008) Guided growth for fixed knee flexion deformity. J Pediatr Orthop 28:626-631

16. Davis RB, Ounpuu S, Tyberski D, Gage JR (1991) A gait data collection and reduction technique. Hum Mov Sci 10:575-587

17. Russell DJ, Rosenbaum PL, Cadman DT, Gowland C, Hardy S, Jarvis S (1989) The gross motor function measure: a means to evaluate the effects of physical therapy. Dev Med Child Neurol 31:341-352

18. Russell DJ, Avery LM, Rosenbaum PL, Raina PS, Walter SD, Palisano RJ (2000) Improved scaling of the gross motor function measure for children with cerebral palsy: evidence of reliability and validity. Phys Ther 80:873-885

19. Novacheck TF, Stout JL, Tervo R (2000) Reliability and validity of the gillette functional assessment questionnaire as an outcome measure in children with walking disabilities. J Pediatr Orthop 20:75-81

20. Palisano R, Rosenbaum P, Walter S, Russell D, Wood E, Galuppi B (1997) Development and reliability of a system to classify gross motor function in children with cerebral palsy. Dev Med Child Neurol 39:214-223 
21. Schwartz MH, Rozumalski A (2008) The gait deviation index: a new comprehensive index of gait pathology. Gait Posture 28:351-357

22. Schwartz MH, Rozumalski A (2008) The gait deviation index: a new comprehensive index of gait pathology. Gait Posture 28:351-357

23. Saraph V, Zwick EB, Zwick G, Steinwender C, Steinwender G, Linhart W (2002) Multilevel surgery in spastic diplegia: evaluation by physical examination and gait analysis in 25 children. J Pediatr Orthop 22:150-157

24. Drummond DS, Rogala E, Templeton J, Cruess R (1974) Proximal hamstring release for knee flexion and crouched posture in cerebral palsy. J Bone Joint Surg Am 56:1598-1602

25. Gage JR, DeLuca PA, Renshaw TS (1996) Gait analysis: principle and applications with emphasis on its use in cerebral palsy. Instr Course Lect 45:491-507 